\title{
AN EXPERIMENTAL INVESTIGATION OF THE CONNEXIONS THE OLFACTORY TRACTS IN THE MONKEY
}

\author{
BY \\ MARGARET MEYER and A. C. ALLISON \\ From the Department of Anatomy, University of Oxford
}

The great expansion of the cerebral cortex which has taken place in higher primates has brought about a considerable displacement of structures on the base of the telencephalon, and the precise comparison of certain areas in this part of the brain with those in lower mammals has been a matter of some difficulty. This is particularly true of the olfactory areas which lie on the orbital aspect of the frontal lobe and the adjacent part of the temporal lobe. Although this part of the brain in primates has been subjected to detailed cytoarchitectural and myelo-architectural examinations (Rose, 1927b, 1928 ; Beck, 1934, and others), the areas directly related to olfaction have never been clearly defined.

It has been generally believed that the olfactory peduncle divides into two main fibre bundles: the lateral olfactory stria, through which fibres pass to the uncus and possibly the hippocampal formation, and the medial olfactory stria, through which fibres reach the subcallosal gyrus, anterior perforated substance, and septum. This specification of the olfactory areas, derived from observations made chiefly in lower mammals with normal material, has not until recently been subjected to experimental verification. As regards the olfactory connexions in lower mammals, Cajal (1901 and 1911) attributed no importance to the medial olfactory stria and denied the existence of any direct olfactory fibres to either the septum or the hippocampus. Recent experimental evidence supports Cajal's point of view. Rose and Woolsey (1943) and Fox and others (1944). stimulated the olfactory bulb in carnivores and recorded potential changes in the prepiriform cortex, the peri-amygdaloid region, and the olfactory tubercle, but none from the septum and hippocampus. Le Gros Clark and Meyer (1947), by using a silver technique for tracing the terminal degeneration in olfactory fibres after ablation of the olfactory bulb in the rabbit, obtained similar results. In addition they observed, in the bed nucleus of the stria terminalis and central amygdaloid nucleus, bilateral degeneration of olfactory terminals appaasently passing through the anterior limb of the anterior commissure. The present study has been undertaken to map out the connexions of the olfactory bulb in the monkey's brain as precise as possible with the same silver technique.

\section{Material and Methods}

Three macaque monkeys (Macaca mulatta) and two immature Guinea baboons (Papio papio) were useid, The operative technique was similar in all cases : ugdest nembutal anæsthesia and with the usual aseptic क्षेe cautions a large right frontal bone flap was reflecter, the frontal lobe of the hemisphere was carefully retraceed, and the olfactory peduncle, lying on the ventral surfigco, was severed. In some cases there were small incident lesions due to contusion on the convexity of the cerbial cortex, but it was possible during the subsequent anatysas to distinguish between the degeneration which resiffied from these lesions and that in the olfactory areas. FKe macaques were allowed to survive four, five, and seve days before decapitation, the baboons three and five days. Brains were removed immediately after dea and fixed in 10 per cent. formol-saline for three weeks They were then divided into blocks of convenient si and a large number of frozen transverse sections were cut, which together formed a complete series through the base of the telencephalon. Most of the sections were impregnated by the ammoniacal silver method of Glees in order to show degeneration of axons and their terminals; other sections taken from the same leves were prepared by the myelin technique of Weil, and also by a Nissl stain, to facilitate identification of the several nuclear masses and fibre systems. In additio complete series of transverse sections through thie normal telencephalon of Macaca, stained by the Weige and cresyl violet methods, were available for study.

In all cases, sections of both sides of the brain were taken and treated identically. Thus, sections of the left or unoperated side of the brain were available for use as controls, and were carefully compared with the corresponding sections of the right side of the braior. In this way, errors which might result from erratio impregnation simulating degeneration were avoided as far as possible. Further, the extent and distribution of degeneration in different cases with different surviva 

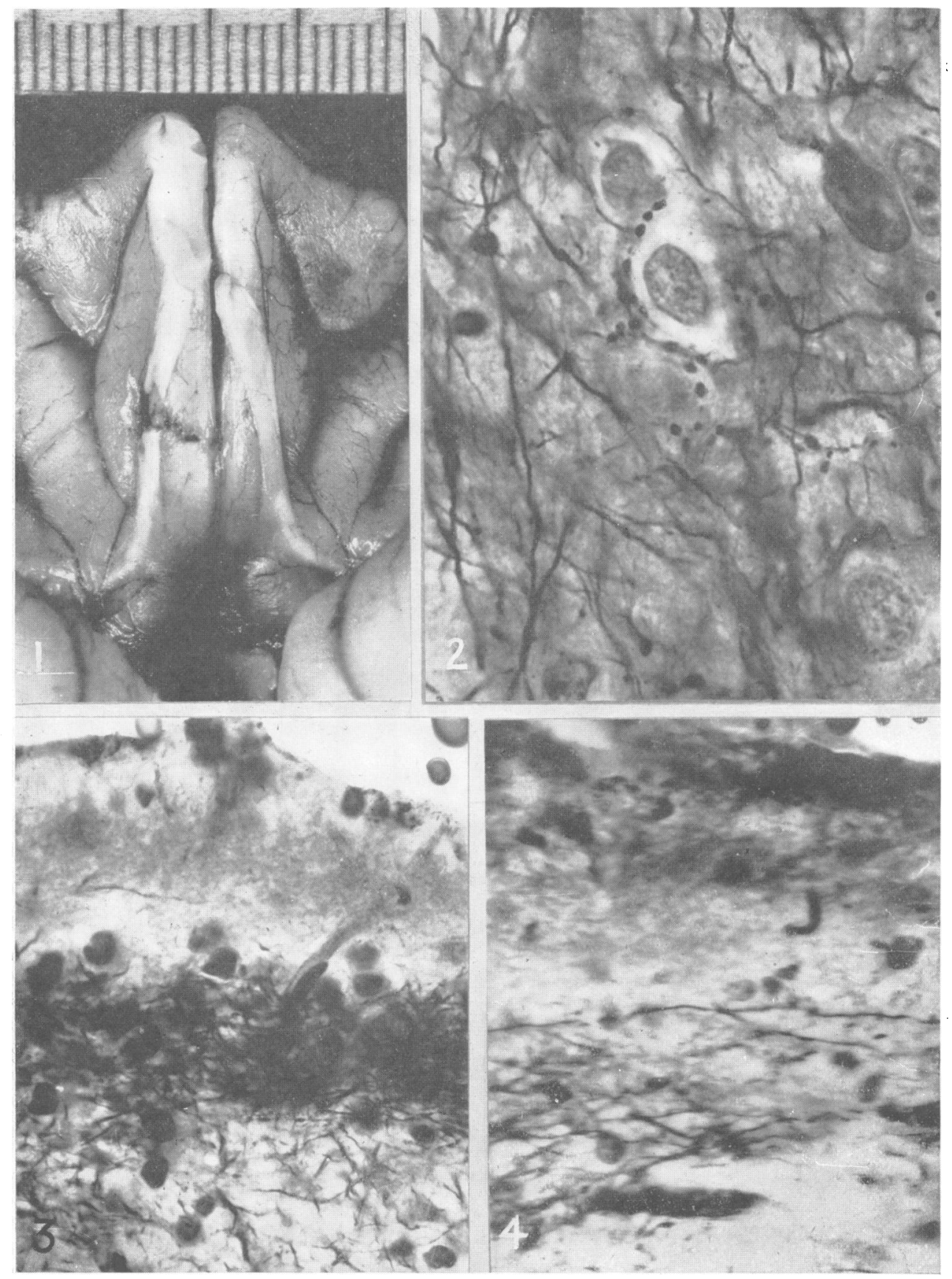

Fig. 1.-Base of frontal lobe of Macaca after operation, showing the right olfactory peduncle transected with very little damage to the gyrus rectus, $\times 2 \frac{1}{2}$.

Fig. 2.-Terminal degeneration around cells of the bed nucleus of the stria terminalis of Papio, five days after transection of the olfactory peduncle. Note that fibres of the stria are intact, $\times 1,100$.

Fig. 3.-Normal olfactory fibres in the superficial layer of temporal prepiriform cortex of Papio, $\times 700$. Compare Fig. 4 , which is the operated side of the same case.

FIG. 4. - Temporal prepiriform cortex of Papio five days after section of the olfactory peduncle, $\times 700$. There is already almost complete degeneration of olfactory fibres, and only brown débris and a few tangential fibres remain. 


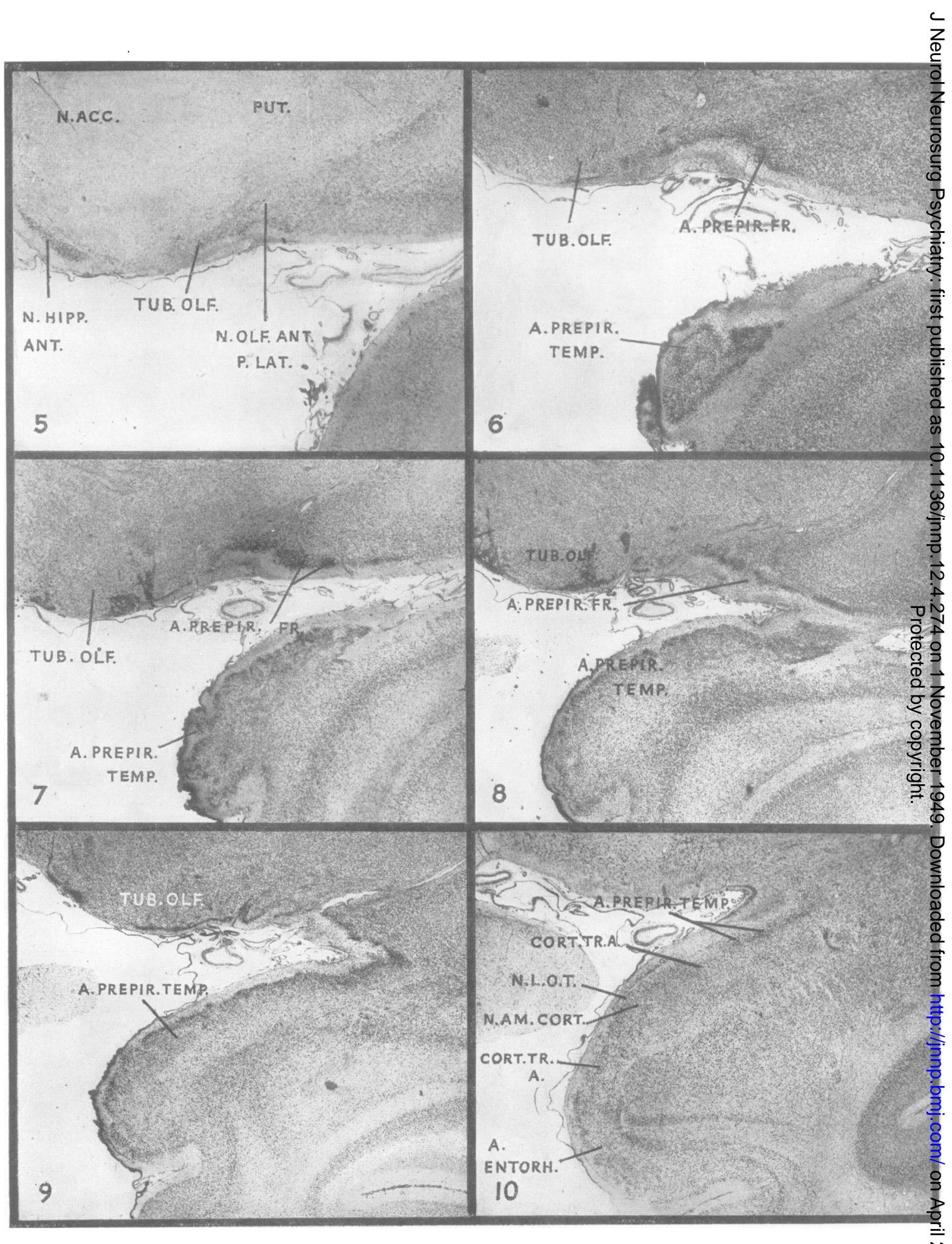

Figs. 5-10.-Representative transverse sections through the olfactory areas of the basal telencephalon of Macack? Nissl stain. All $\times 10$ except Fig. 6 which is $\times 13$. In Fig. 10 the posterior extremity of the temporal prepiriforpo area (which goes over into medial amygdaloid nucleus) and of the dorsal part of the cortico-amygdaloid transitiog area are shown. 
periods were found to be very consistent. The early stages of degeneration are recognizable chiefly in the terminals and the very fine preterminal fibres; later, these are entirely disintegrated and the coarser fibres break up. By thus having a series of cases showing different stages of degeneration, it is possible to reconstruct fairly accurately the course and termination of the fibre pathways involved.

\section{Observations}

The course and distribution of degenerative changes following sectioning of the olfactory peduncle will be described by reference to a series of transverse sections through the telencephalon of Macaca, the levels of which are shown in Fig. 17. The results of experiments on Papio are so similar to those on Macaca that no separate description is necessary.

In general, we have followed the terminology used by Crosby and Humphrey (1941) for the corresponding parts of the human brain, and that used by Lauer (1945) for the macaque brain, although our interpretation of the homologies of certain regions differs in some respects from theirs. Most of the

\section{Abbreviations Used in All Figures}

a. entorh.

entorhinal area.

a.preop.med.

a.prepir.fr.

a.prepir.temp.

caps.int.

ch.opt.

claust.

com. ant.

com.ant.l.ant.

cort. tr.a.

forn.

lim. ins.

n. acc.

n.am.b.a.

n.am.b.l.

n.am.b.m.

n.am.cent.

n.am.cort.

n.am.med.

n. caud.

n.diag.b.

n.hipp.ant.

n.l.o.t.

n.olf.ant.p.lat.

n.sept.lat.

n.sept.med.

n.str.term.

pall.

put.

sulcus entorh.

sulcus rh.

tr.op.

tub.olf.

vent. lat.

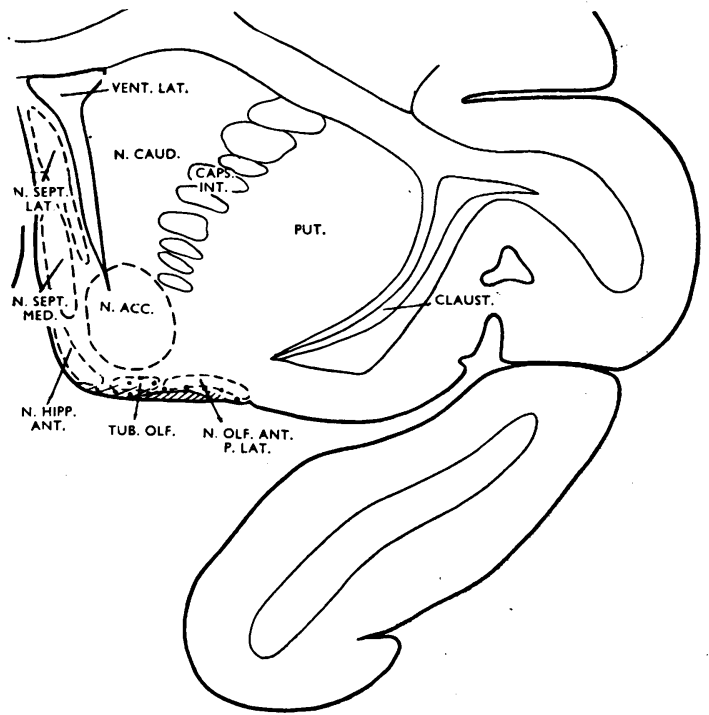

Fig. 11.-Outline of transverse section through the cerebral hemisphere of Macaca at the level of the septum and the anterior margin of the olfactory tubercle. In this and the other text figures, degeneration of terminals is indicated by dots, and that of fibres by hatching.

primary olfactory areas are illustrated in the Nissl sections shown in Figs. 5 to 10. As far as the cortex of the piriform lobe is concerned, we have not been able to apply directly the cyto-architectonic subdivisions described in different species of mammals by Rose (1927a and b, 1928). Following the recommendation of Brodmann (1909) and others, we have recognized only two cortical areas in the anterior part of the hippocampal gyrus, the prepiriform area and the entorhinal area (Fig. 17). Also, in accordance with the current terminology, we have included the so-called peri-amygdaloid area, which has been regarded by some authorities as part of the piriform lobe cortex, in the amygdaloid complex.

Level 1 (Figs. 5 and 11).-At this transverse level the olfactory tract has already deviated somewhat laterally from the position it occupies further forward where the olfactory peduncle joins the orbital surface of the hemisphere. It is made up, of a flattened dense mass of fibres which are obliquely cut, so that it is not possible to recognize the different component parts of the tract described by Lauer (1945) and others. The tract overlies the deeply-stained cells of the anterior olfactory nucleus, the lateral part of which grades laterally into neocortex. Only a very slight depression on the surface of the brain, which corresponds to the anterior extremity of the rhinal fissure in lower 


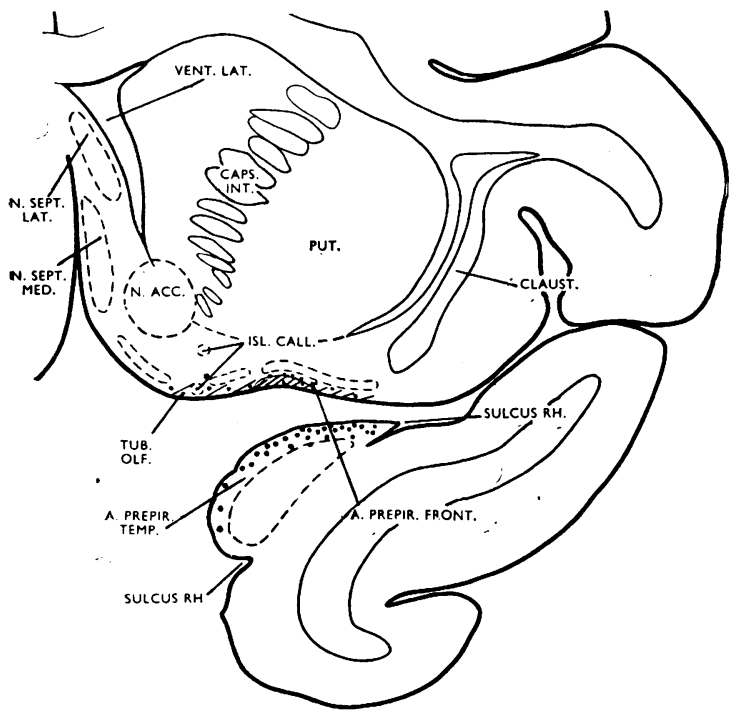

Fig. 12.-Transverse section at the level of the olfactory tubercle and the anterior margin of the prepiriform area.

mammals, marks this transition. On the medial side of the tract the medial part of the anterior olfactory nucleus has been replaced by the rostral extremity of the olfactory tubercle, and the latter is adjoined by the large cells of the anterior hippocampal nucleus which reach upwards towards the septal nuclei. A medial fascicle from the olfactory tract is clearly defined, extending as far as the superficial layer of the anterior hippocampal nucleus. A smaller lateral fascicle lies superficial to the lateral part of the anterior olfactory nucleus ; it does not extend as far laterally as the isocortex.

Seven days after transection of the olfactory peduncle, many, but not all, fibres of the lateral olfactory tract are degenerated. There is almost complete disintegration of the axons in the lateral and medial fascicles, and even under low magnification a definite loss of fibres is apparent when the section is compared with that of the normal side. Degenerating fibres of the medial fascicle can be traced into the superficial layer of the anterior hippocampal nucleus, but fibres among the cells of the nucleus are intact. Some degeneration is seen in that part of the anterior olfactory nucleus which lies immediately above the olfactory tract, and which appears to be equivalent to the pars externa of the anterior olfactory nucleus in lower mammals. Otherwise the fibre plexus within the anterior olfactory nucleus is normal.

In the three-day experiment very little change is seen in the fibres of the tract, but degeneration of terminals is more distinct. It is most striking in the olfactory tubercle, where there are numerous disintegrating fine fibres and enlarged termina boutons in the superficial layer; these extend inte the pyramidal cell layer, and some penetrate as deeply as the polymorph layer (particularly in the vicinity of the olfactory tract). Further medially degenerative changes are again confined to the superficial layer of the anterior hippocampar nucleus; only in one case, complicated by a incidental lesion in the cerebral cortex, was degeneration found among the cells of the nucleus itself. The septal nuclei, including the nucleus? accumbens, remain entirely normal.

Level 2 (Figs. 7 and 12).-At this level, the laterat fascicle of the olfactory tract is much more extensive. and is accompanied by the deeply-stained band of pyramidal cells of the prepiriform cortex, which has replaced the lateral part of the anterior olfactors nucleus. The olfactory tubercle is enlarged cons siderably and shows well-defined lamination close to the tract, while more medially the islands oft Calleja are very conspicuous, sometimes lying close to the surface of the brain. The short medin fascicle of the olfactory tract ends before the mefiat part of the tubercle is reached. The rostral $\mathrm{fx}^{2}$ tremity of the prepiriform area on the tempबraI lobe is limited on both sides by the curved antero part of the rhinal fissure. In silver preparatigne the first layer of the prepiriform area consists dense mass of fine fibres which turn inwards tow the pyramidal cell layer, becoming less numeralus

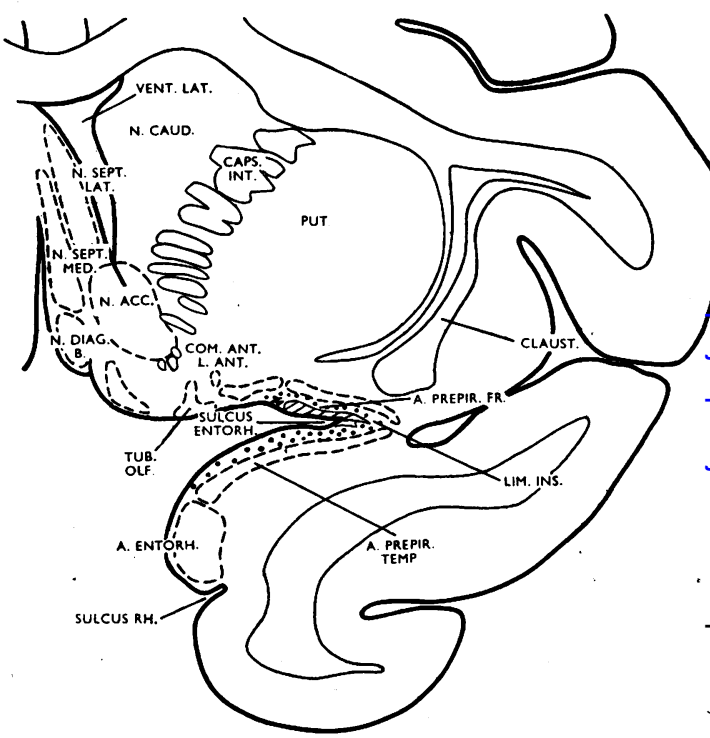

Fig. 13.-Transverse section at the level of the limeno insulæ. 
on either side as the rhinal fissure is approached. In Nissl sections this area is characterized by its darkly stained, slightly corrugated band of pyramidal cells overlying the isocortex of the temporal lobe (Figs. 6 and 7).

Degeneration at this level is best seen in the fourand five-day experiments. It is particularly marked in the superficial layers of the prepiriform cortex of the temporal lobe, the fine fibres of which show almost complete drop-like disintegration. The extent and distribution of this degeneration corresponds precisely to that of the penetrating fibres just mentioned as occupying the superficial cortical layer of the normal side. Isolated degenerating fibres pass into the pyramidal cell layer. The degeneration in the prepiriform area of the temporal lobe is considerably more severe than in the prepiriform area on the orbital surface of the frontal lobe. In the latter region the lateral fascicle is beginning to break up and terminal degeneration is again confined to the superficial and plexiform layers. The lateral part of the tubercle also shows in its plexiform layer a large number of disintegrating terminals. Some of these penetrate into the deeper layers of the tubercle and into the more superficially situated islands of Calleja. The medial part of the tubercle, the septal nuclei, and the nucleus accumbens are all normal.

Level 3 (Figs. 8 and 13).-At this level the frontal lobe becomes connected with the temporal lobe through the limen insulæ. From the tract a large component passes to the junction of the frontal and temporal lobes in the entorhinal sulcus and is continuous with the dense fibre feltwork in the superficial layer of the adjacent part of the prepiriform area of the temporal lobe (Fig. 3). Thus, even normal preparations make it apparent that the finer fibres in the superficial layer of this part of the prepiriform area are formed by the terminal arborizations of axones of the lateral olfactory tract. Some fibres of the lateral olfactory tract spread across towards the insula, accompanied by the pyramidal cell layers of the prepiriform cortex of the frontal and temporal lobes. The cytoarchitectural features of these areas are clearly seen in Nissl preparations (Fig. 8). The lateral olfactory tract also contributes a short medial fascicle to the olfactory tubercle. The anterior limb of the anterior commissure is seen to consist of three small bundles of fibres situated close to the tubercle and ventro-lateral to the nucleus accumbens:

The extent and distribution of degeneration at this transverse level is well seen four and five days after section of the olfactory peduncle. Examination with low power reveals a marked difference in intensity of staining between olfactory fibres in the normal and operated sides. Fibres on the normal side are stained a deep black, while those on the side of lesion are a much lighter brownish colour. Under high magnification an unbroken band of degenerating fibres and terminals is seen in the superficial and plexiform layers of the prepiriform cortex, extending from the lateral third of the orbital surface of the frontal lobe as far as the limits of the entorhinal area on the dorsal surface of the temporal lobe. In the entorhinal area itself only very few, isolated, degenerating fibres are seen at its margin of contact with the prepiriform area. In both these experiments with four and five days' survival after operation many of the thick fibres of the olfactory tract itself are still intact, whilst the fine terminal fibres in the superficial cortical layers of the temporal lobe all show drop-like disintegration. This confirms previous observations that terminal arborizations of axones degenerate some time before the axones themselves are affected. The fascicle of the olfactory tract which spreads towards the insula is degenerated, but it does not actually reach the isocortex of the insula, and there is no evidence of degeneration in any of the cortical layers of the insula itself. Compared with more anterior levels, the tubercle receives few olfactory fibres, and these are localized to the part nearest the olfactory tract. The septal nuclei and nucleus accumbens are again normal. The bundles of the anterior limb of the anterior commissure are cut transversely, and hence it is not possible to detect with certainty any degeneration in them.

Level 4 (Fig. 14).-At this level the lateral olfactory tract can no longer be observed macroscopically on the surface of the brain. In sections the main bulk of the tract is seen to have swung around to terminate in the rostral part of the prepiriform area on the temporal lobe, as described in the preceding levels. The remainder of the lateral olfactory tract, much reduced in size and cut somewhat obliquely, lies in the entorhinal sulcus, and a fascicle (cut longitudinally) passes from the main tract along the superficial layer of the prepiriform area of the temporal lobe. The pyramidal cell layer of the prepiriform cortex is adjoined ventro-medially by the cortical transition area, which overlies the foremost extremity of the basal accessory amygdaloid nucleus.

In the four- and five-day experiments most of the fibres of the lateral olfactory tract in the angle between the frontal and temporal lobes are in process of degeneration, and the superficial layer of the prepiriform cortex on the dorsal surface of the temporal lobe has much the same appearance 


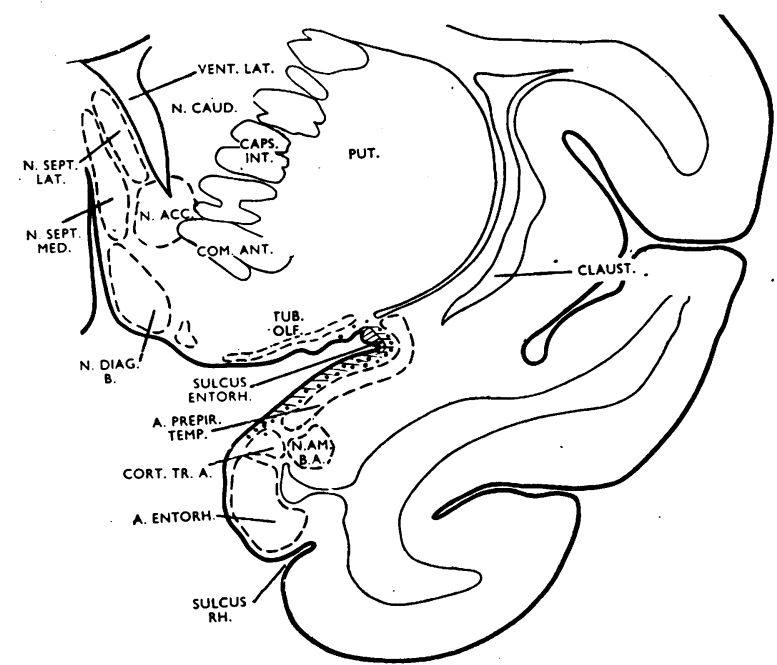

Fig. 14.-Transverse section at the level of the anterior margin of the amygdaloid formation.

as in the preceding level. Degenerating fibres in the superficial layer of the cortex terminate as far ventrally as the transition area. The entorhinal area is quite normal. The distribution of olfactory fibres to the tubercle is insignificant at this level, only the most lateral part of this region, adjacent to the lateral olfactory tract, being affected. More medially, the superficial fibres which surround the tubercle are normal, and appear to be derived from the most ventral fibres of the external capsule.

The seven-day case shows a severe loss of fibres in the superficial and plexiform layer of the prepiriform cortex when compared with the normal side, and in those fibres which are left degeneration is far advanced (Figs. 3 and 4). Some normal fibres remain, which are probably tangential fibres arising (according to Cajal) from nerve cells in the first cortical layer. Again, as in the rabbit, degeneration is confined to the outermost and plexiform layers of the prepiriform cortex, thus indicating an axo-dendritic contact between olfactory terminals and cells of the piriform cortex. There is no evidence of fibre or terminal degeneration elsewhere at this level.
Level 5 (Fig. 15). -This level corresponds $\stackrel{\mathbb{Q}}{\subset}$ to the most caudal part of the crossing of the anterior commissure. The prepiriform $\stackrel{\text { ? }}{=}$ cortex has been replaced by the cortico-mediak group of amygdaloid nuclei, and the various ${ }_{\omega}^{T}$ elements of the latter can here be mapped $\delta$ out clearly. The lateral olfactory tract is $\frac{\mathrm{N}}{\mathrm{N}}$. much reduced in size, its fibres occupying a similar position to that described in the preceding level. The fascicle passing from the main tract now surrounds the cortico-o medial amygdaloid group. The bed nucleus of the stria terminalis has reached its greatest $\frac{\bar{w}}{\overline{5}}$ extent, beginning at the dorso-medial aspect $\stackrel{\varnothing}{\Omega}$ of the internal capsule, near the caudates nucleus, and passing around the caudal part ${ }^{\mathrm{s}}$ of the anterior commissure to merge ventrally? with the medial preoptic region.

Four and five days after section of theo olfactory peduncle, degenerating fibres of the lateral olfactory tract spread into the mediato amygdaloid nucleus, and fine terminal fibres $\vec{v}$ showing drop-like disintegration surround itst nerve cells. That part of the nucleus which पpर close to the tract receives more olfactory fibfos than the lateral part, where the fibre plexus ㅇํㅇ almost intact. In the peri-amygdaloid fascicle $\mathrm{Bf}_{7}^{-}$ the tract, degeneration is very marked. A numbro of fibres terminate in both parts of the nucleus the lateral olfactory tract, only the posterior part of which is shown in Fig. 15. Degeneratiog fibres and terminals remain in the superficito

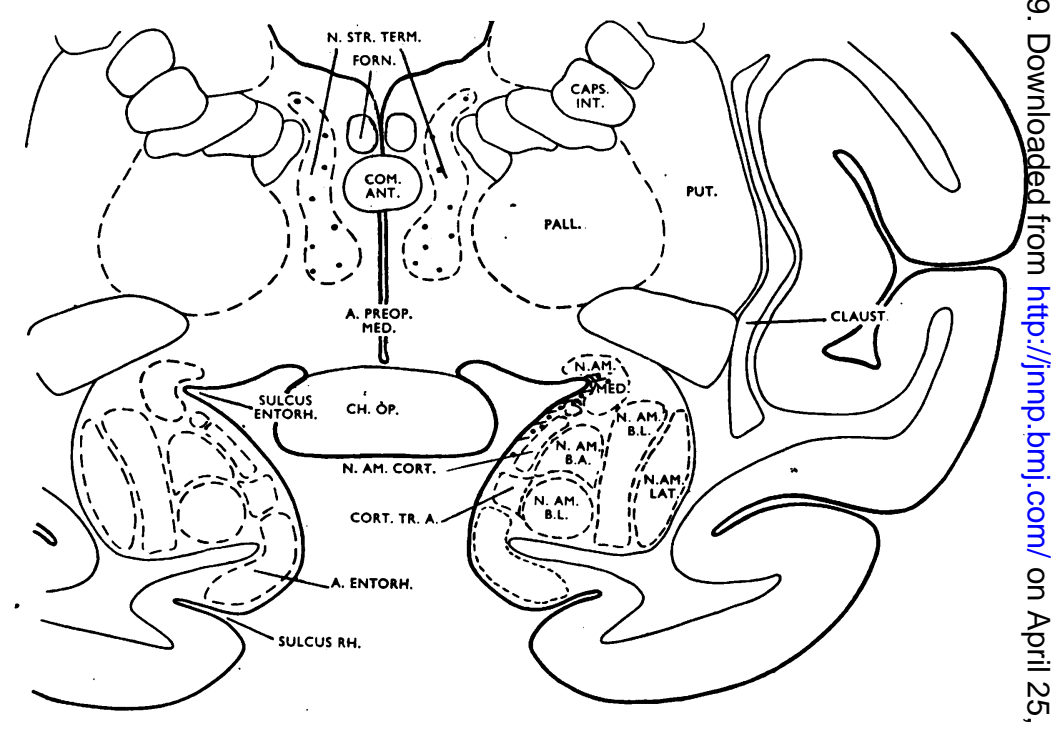

FIG. 15.-Transverse section at the level of the optic chiasma and the middle $\stackrel{N}{\sim}$ of the amygdaloid formation. 


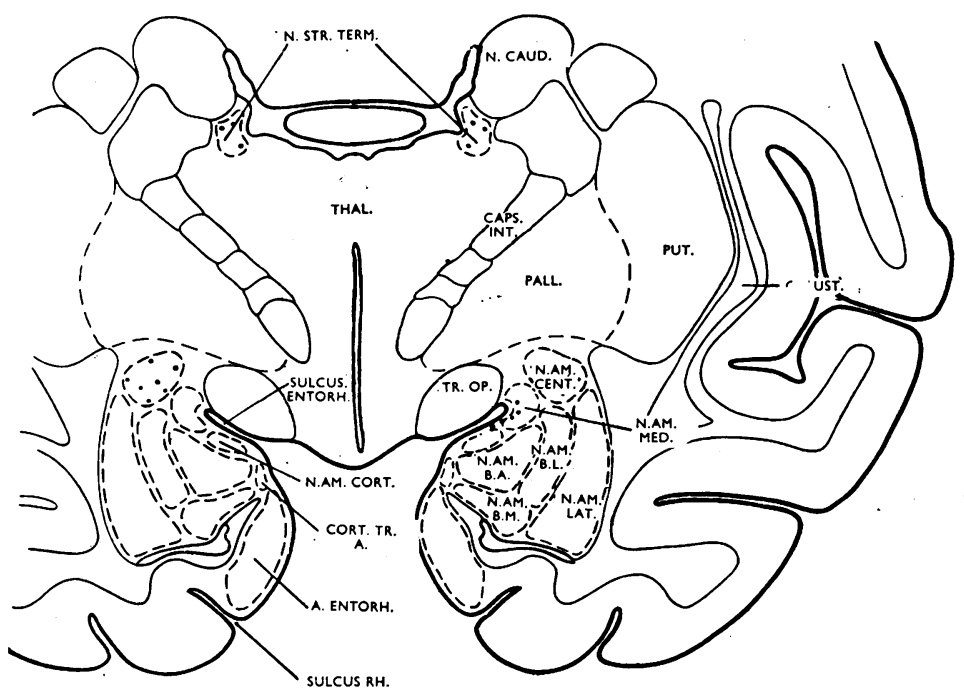

FIG. 16.-Transverse section at the level of the caudal part of the amygdaloid formation.

region and $T$ nucleus of the stria terminalis are affected bilaterally. The fibre plexuses of the lateral preoptic region, hypothalamus, and other areas are intact.

Level 6 (Fig. 16).-This level corresponds approximately to the posterior third of the amygdaloid complex. The central amygdaloid nucleus has now appeared and the baso-lateral group has expanded at the expense of the cortico-medial group of nuclei. The nucleus of the stria terminalis lies between the caudate nucleus and the thalamus.

Olfactory fibres and terminals become progressively more sparse posteriorly, so that at this level in the four- and fiveday experiments degeneration

layer of the cortical amygdaloid nucleus and make no contact with the cell bodies of the nucleus. They can be traced as far as the transition area. The entorhinal area is apparently quite unaffected.

There is bilateral terminal degeneration in the nucleus of the stria terminalis. Much of the fibre plexus within the nucleus has remained intact, but a number of fine, degenerating, terminal fibrils surround the nerve cells of the nucleus in typical drop-like disintegration (Fig. 2). The fibres of the stria terminalis itself are normal. In the seven-day experiment the sections have been cut slightly more caudally. The bed nucleus of the stria terminalis appears as two circumscribed masses of small cells, the larger in the medial preoptic region and the other, which goes over eventually into the central amygdaloid nucleus, between the caudate nucleus and the anterior extremity of the thalamus. Few degenerating fibres are seen just medial to the internal capsule, but the severity of degeneration increases as the nucleus of the stria terminalis in the medial preoptic region is approached. The boundaries of the nucleus are not very distinct, and signs of degeneration appear to extend into the periventricular region and into what is usually considered a part of the medial preoptic nucleus. In this case, too, the preoptic in the medial and cortical amygdaloid nuclei is much less severe than in the preceding level. In the bed nucleus of the stria terminalis and the central nucleus there is bilaterally a breaking-up of some of the fine intrinsic fibres. The other amygdaloid nuclei, the entorhinal area, and the hypothalamus show no signs of degeneration.

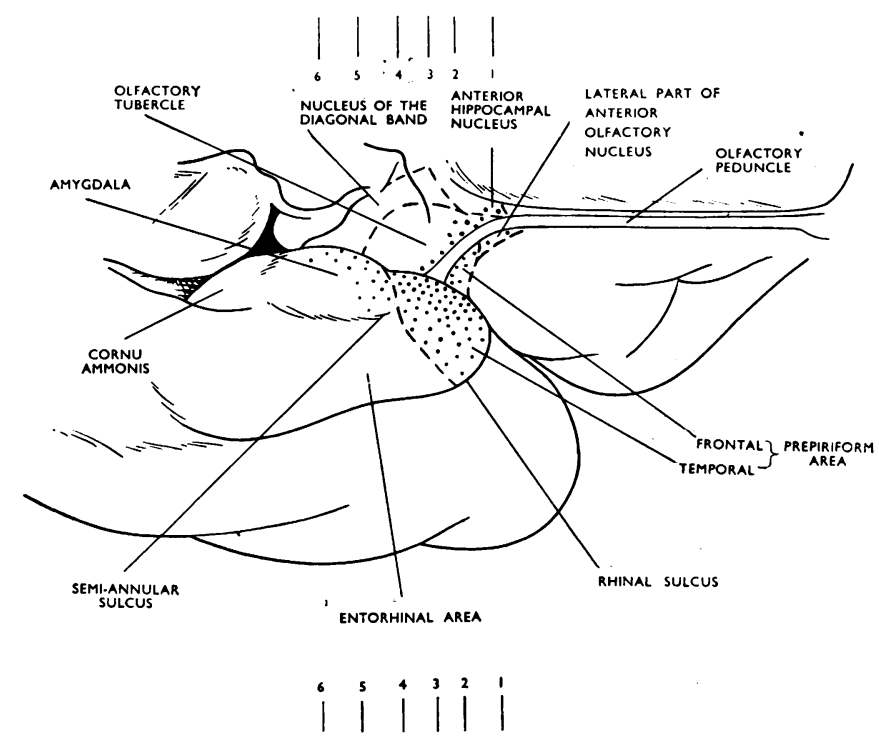

FIG. 17.-The basal telencephalic areas of Macaca, with the temporal lobe somewhat retracted to display the distribution of olfactory terminals (dotted). 


\section{Discussion}

The experimental evidence presented in the preceding pages enables us to give a more accurate account of the central connexions of the olfactory tracts in the monkey's brain than has previously been possible. Before discussing the different areas of termination separately, we propose to recount the course of fibres of the olfactory tracts according to these observations. In most cases the olfactory peduncle was severed just before it fuses with the orbital surface of the frontal lobe (Fig. 1), and thus fibres which the anterior olfactory nucleus may contribute to the olfactory tract had also been interrupted. As the lateral olfactory tract continues caudally, a medial and a lateral fascicle are given off. The medial fascicle extends into the plexiform layer of the anterior hippocampal nucleus, but it does not reach the septal nuclei or the isocortex of the gyrus rectus, as has been suggested by Lauer (1945) from normal preparations. The lateral fascicle terminates in the superficial layer of the frontal extension of the prepiriform cortex, not extending as far as the neocortex of the orbital gyri (as it is also stated to do by Lauer).

As the olfactory tubercle is approached the lateral olfactory tract becomes wider. Its most medial fibres terminate in the rostral and lateral part of the olfactory tubercle, but the great majority deviate laterally towards the limen insulæ, skirting the anterior perforated space. As in the rabbit, we have not been able to distinguish a bifurcation of the tract, with the formation of a separate "medial root" to the septal region in the macaque brain. Different components of the tract can be distinguished within the olfactory peduncle, but at the more caudal levels no clear division into roots is recognizable. Reaching the limen insulæ, the lateral olfactory tract disappears from the surface of the brain. In silver sections, however, it is seen that at this point the terminal arborizations of the great majority of fibres (not visible in myelin preparations) are folded over and extend forward over the dorsal surface of the temporal lobe to end in the prepiriform cortex which forms the anterior part of the hippocampal gyrus (gyrus ambiens in man). Thus at some transverse levels (for example see Fig. 12) the olfactory tract itself is seen on the orbital surface of the frontal lobe and (in the same section) its terminations on the temporal lobe. Some fascicles continue caudally in the entorhinal fissure to the peri-amygdaloid region (or gyrus semilunaris), where they terminate in the superficial nuclei of the cortico-medial group of the amygdaloid complex. The anterior limb of the anterior commissure (or " intermediate olfactory stria ") appears to be the pathway of olfactory fibres to the bed nucleus of the stria terminalis, which extends ato one end into the preoptic region and at the othero to the central amygdaloid nucleus.

Olfactory Tubercle.-There has been some divergence of opinion whether the olfactory tubercles received fibres from the olfactory tract (Cajal, 1901 ; Elliot Smith, 1909, and others). Recently Le Gros Clark and Meyer (1947) demonstrated that extirpa-r. tion of the olfactory bulb in rabbits is followed by $\vec{F}$ terminal degeneration in the olfactory tubercle, $\mathrm{a} \stackrel{\oplus}{+}$ finding which supports the experimental physio-? logical evidence of Fox and others (1944) in the cat. In our experiments, sectioning of the olfactory $\frac{\bar{D}}{\sigma}$ peduncle in the monkey was followed by degenera- 2 tion in the anterior and lateral parts of the tubercle. It was found that, as in the rabbit, fibres terminate mainly in the plexiform layer, although somepenetrate into the deeper layers of the tubercle and $\vec{\omega}$ in a few instances into the superficial islands of Calleja. Rose (1927a and b), on the basis of differences in cyto-architecture, divided the olfactory. tubercle into rostral, middle, and caudal regions, $\vec{N}$ Tol 1, Tol 2, and Tol 3. According to his detailed comparative studies, the rostral part, although weth- $-\mathrm{A}$ developed in lower mammals, is rudimentary structure in the monkey and in Man. In the rabbe his area Tol 1 is more extensive and corresponas $Z$ closely with the part receiving olfactory fibres, determined by Le Gros Clark and Meyer. In $\mathrm{t} B \mathrm{e}_{3}^{\Phi}$ present investigation we have found that Roses delimitation of the rudimentary area Tol 1 in the monkey again coincides almost precisely with o풍 definition of the area in which olfactory fibres $\overrightarrow{0}$ terminate. An interesting demonstration of theo close correspondence between cyto-architecture and function is thus afforded for the olfactory area of the tubercle. From the reduced extent of this area it is evident that in the monkey the tubercle partici-市 pates in olfaction only to a limited degree, and its $\stackrel{2}{\Rightarrow}$ quite considerable size in this species is thus evidently을 due to the relative enlargement of the non-olfactory areas.

Amygdaloid Complex.-Johnston (1923), on the basis of differences which he observed in comparative studies, divided the amygdaloid complex into a cortico-medial and a baso-lateral group. Subsequent workers have followed his classification, 0 recognizing in addition an anterior amygdaloid area and a cortico-amygdaloid transition area. In theiro description of the amygdaloid complex in the human brain, Crosby and Humphrey (1941) state that the baso-lateral group of nuclei is more highly differentiated than in macrosmatic mammals, while the cortico-medial group (in particular the nucleus of 0 the lateral olfactory tract and the medial and central 
amygdaloid nuclei) is much reduced. This observation suggested to these authors a relationship in function and in degree of development between the cortico-medial group and the main olfactory centres.

In rabbit experiments, Le Gros Clark and Meyer (1947) showed that the cortico-medial group of the amygdaloid complex receives direct fibres from the olfactory bulb, but the baso-lateral group does not, a fact which demonstrates that Johnston's two subdivisions are functionally as well as phylogenetically distinct. After sectioning of the olfactory peduncle in the monkey, the pattern of distribution of olfactory fibres to the amygdaloid complex is essentially the same as in the rabbit, although the position of the areas which are wholly or partly olfactory in function is somewhat altered and they are relatively much reduced in size. In lower mammals the superficially placed nuclei of the cortico-medial group, often termed the periamygdaloid area, lie on the basal surface of the telencephalon and constitute, together with the prepiriform cortex which lies dorsolateral to the peri-amygdaloid region, the anterior part of the piriform lobe. With the enlargement of the neocortex in monkey and man the peri-amygdaloid region is moved forward in a dorso-medial direction and comes to lie on the dorsal surface of the temporal lobe, where it forms the gyrus semilunaris. Thus the prepiriform cortex now lies anterior to the peri-amygdaloid area and the sulcus semiannularis separates the two regions.

Most of the fibres of the lateral olfactory tract end anterior to the peri-amygdaloid area, and, in the monkey, only a small component passes backwards in the entorhinal sulcus to spread over and terminate in the peri-amygdaloid region.* After sectioning the olfactory peduncle in Macaca and Papio degeneration of this component of the lateral olfactory tract shows that its fibres are distributed mainly to the antero-medial part of the periamygdaloid nuclei. Olfactory fibres penetrate quite deeply into the medial amygdaloid nucleus, apparently terminating in contact with the bodies of the neurones of which it is composed. The nucleus of the lateral olfactory tract consists of two parts, as has been described for man, the rabbit, and the bat. Fascicles of degenerating fibres detach themselves from the olfactory fasciculi in the superficial layer of the peri-amygdaloid region to enter this nucleus and terminate on its nerve cells. Part of the nucleus lies amongst the olfactory fibres which pass on in the superficial layer (Fig. 10). The cortical amygdaloid nucleus is more highly differentiated in the

\footnotetext{
* Rose (1927) did not recognize in his cyto-architectural studies that the lateral olfactory tract together with the prepiriform cortex has been pushed further forward than the amygdaloid region for he states that the entire tract terminates in the area peri-amygdalaris.
}

monkey and in Man than in lower mammals and has been regarded by several authors as true cortex. According to the evidence presented here it receives fewer olfactory fibres than the medial amygdaloid nucleus or the nucleus lateral olfactory tract. Degenerating fibres and terminals are localized in the superficial and plexiform layer of the cortical nucleus, which indicates that synaptic contact is axodendritic, as in the prepiriform cortex.

Lauer (1945), in describing the amygdaloid nucleus in the brain of Macaca, places the cortico-amygdaloid transition area in the lateral part of the amygdaloid fissure between the peri-amygdaloid region and the entorhinal area (which is labelled piriform cortex in his diagrams). We could not certainly trace any olfactory fibres to this region. In the material at our disposal another group of nerve cells could be identified, lying in the anteromedial part of the semi-circular amygdaloid fissure (or semi-annular sulcus). It forms a transition area between the prepiriform cortex and the periamygdaloid region (Fig. 10) and is also in cellular continuity with the anterior part of the basal accessory amygdaloid nucleus. A substantial component of olfactory fibres was traced into this area. It is possible that this is the antero-medial part of a transition area which lies under the entire semiannular sulcus and is continuous with the corticoamygdaloid transition area described by Lauer in the macaque and also by Crosby and Humphrey in the human brain. Only the antero-medial part of it, however, receives olfactory impulses and perhaps functions, as Crosby and Humphrey suggested, as a distributing centre for olfactory impulses to the baso-lateral group.

Olfactory fibres are distributed to the two remaining nuclei of the cortico-medial group, namely the bed nucleus of the stria terminalis and the central amygdaloid nucleus, by way of the anterior limb of the anterior commissure. These two nuclei are directly continuous, the bed nucleus of the stria terminalis extending from the amygdaloid region upwards and forwards as a thin band over the internal capsule and then reaching downwards into the medial preoptic region, where it attains a considerable size (as Papez and Aronson, 1934, point out). After transection of the olfactory peduncle degeneration in both these nuclei is bilateral. In the monkey, however, a substantial part of the fine fibre plexus remains intact, whereas in the rabbit there is almost complete degeneration of the terminal plexus after excision of the olfactory bulb. The different results in the two species suggest that in the monkey the central amygdaloid nucleus and the bed nucleus of the stria terminalis are not predominantly olfactory in function, as they 
evidently are in the rabbit. The regression of the olfactory component of the anterior commissure in primates, which has been emphasized by Fox and others (1948) in an experimental Marchi study, supports this inference. Nevertheless, our material provides convincing evidence that even in primates the bed nucleus of the stria terminalis (particularly that part which lies in the preoptic region) and the central amygdaloid nucleus form a definite part of the olfactory system. Physiological evidence in accordance with this observation has been obtained in cats by Hess and Müller (1946), who report centrally induced sniffing responses after electrical stimulation of the nucleus of the stria terminalis and other related areas.

Prepiriform Cortex.-The great majority of fibres of the lateral olfactory tract terminate in the superficial layers of the prepiriform cortex. The method which we have employed has enabled us to map out precisely the extent of the degenerating olfactory terminals, and in Fig. 17 their distribution is indicated on the surface of the brain. This prepiriform area, which represents the primary olfactory cortex, has never been clearly defined in primates; previous investigators have failed to homologize it with the relatively much larger region it occupies in lower mammals, even though the cyto-architecture is typical. Phylogenetically, the folding over and displacement of the so-called rhinencephalon in a fronto-medial direction begins in lower primates with the development of the neocortex of the temporal lobe. The posterior part of the hippocampal gyrus (the entorhinal area) is also gradually moved forward and the prepiriform cortex (together with the terminal arborizations of the lateral olfactory tract) is folded over the frontal lobe and displaced in front of the uncus. In the macaque and baboon, the primary olfactory cortex thus lies on the dorsal surface of the temporal lobe. It occupies the anterior part of the hippocampal gyrus (which corresponds to the gyrus ambiens in man), and it is clearly separated from the neocortex of the temporal pole by the rhinal sulcus, while the sulcus semi-annularis delimits it from the amygdaloid complex of the gyrus semilunaris. The temporal prepiriform cortex passes over without any external markings into the entorhinal area, whereas in man a sulcus separates the gyrus ambiens from the hippocampal gyrus proper. In normal silver preparations the first layer of the temporal prepiriform cortex consists of a dense mass of fine fibres turned inwards towards the pyramidal cell layer. These fibres degenerate after sectioning of the olfactory peduncle, and, since signs of terminal degeneration are rarely seen within the pyramidal cell layer, it is concluded that synaptic contact is axo-dendritic. No olfactory fibres reach the iso-ồ cortex of the insula directly. Beck (1934), in a? description of the myelo-architecture of the dorsalo cortex of the temporal pole in the monkey, describes ${ }_{\infty}^{D}$ the first layer of this region as extraordinarily thick, increasing in thickness antero-posteriorly (that is to say as it approaches the olfactory tract). His description corresponds to our findings, but he fails to relate the fibre layer he describes to theor olfactory tract and does not appear to realize thato these fibres are its terminal arborizations. Theo dense superficial fibre layer coincides with the $\frac{\bar{p}}{\frac{1}{5}}$ appearance of typical prepiriform cortex, the cyto- $\mathbb{\mathbb { Q }}$ architecture of which in the macaque is closely@ homologous with that of lower mammals. It is characterized by its well-defined second layer of? closely packed pyramidal cells which are sometimes $\overrightarrow{\vec{\omega}}$ grouped into islets. The series of Nissl-stainedo sections through this region of allocortex (shown in Figs. 5 to 10) is representative of this area in the? macaque.

The area of prepiriform cortex which lies on the orbital surface of the frontal lobe is very restrictedin in primates; it appears somewhat behind the rostral limits of the olfactory tubercle and recei正? relatively few olfactory fibres. This area occupes the same general position as does the correspondiago area in the rabbit and mouse.

Rose (1927 a and b) has made an extensive studigy of the cyto-architecture of the allocortex in lowere mammals and in primates. According to interpretation the prepiriform cortex in the monkeyt (Cynocephalus hamaaryas) is restricted to the basat surface of the frontal lobe (his prepyriform areas 1 , 2 , and 3) with the exception of a very small part of prepyriform 3, which overlaps a little on to the temporal lobe. The temporal prepiriform or primary olfactory area, localized from our experi $\frac{\Omega}{-}$ mental material, is included in his diagrams of the $\overrightarrow{0}$ monkey in the entorhinal region (the anterior pare of areas e $\alpha, e \beta, e v)$. However, his photomicrograph of these areas has been taken at the level of the. amygdaloid nucleus, where the cortex is different: from the anterior part and clearly belongs to the non-olfactory entorhinal area. In lower mammals, also, Rose has not distinguished clearly between the prepiriform cortex, the entorhinal area, and the peri-amygdaloid region, in some instances regarding the whole olfactory cortex of the piriform lobe as peri-amygdaloid area (lemur, dog), in others as part of the entorhinal area (mouse). In a later como parative study on the insular cortex (1928) Rose includes the temporal prepiriform cortex of the monkey in the agranular cortex of the insuld 0 (ai 9, ai 10). The cyto-architecture is described in 
detail by him and confirms our own evidence; he makes no suggestion, however, that this area is to be regarded as prepiriform cortex. He does indicate that the entire agranular insular cortex might have functions related to smell ; but, in his definition of agranular insular cortex, areas are included which have certainly no connexion with olfactory fibres. In Brodmann's cyto-architectural charts of lower mammals, the prepiriform cortex (together with the olfactory tubercle and peri-amygdaloid region) is included in area 51. In his maps of the lemur and monkey cortex this region is unlabelled. Area 34 in Brodmann's chart of the human cortex appears to be equivalent to the prepiriform cortex, together perhaps with the anterior part of his area 28 (entorhinal area). There is, as yet, no precise definition of the extent of the cortical projection area of the olfactory tract for the human brain. Brodal's (1947) attempt to transfer the findings in animals to the brain of man is based mainly on the descriptions and figures of Rose, but it is doubtful whether the functional properties of any cortical fields can be defined solely by their architectonic features, without reference to their connexions. Lauer (1945) includes the prepiriform area of Macaca in his lateral part of the anterior olfactory nucleus.

Physiological evidence supports the view that the primary olfactory area in monkeys is located in the anterior extremity of the hippocampal gyrus. As early as 1886 Ferrier reported that electrical stimulation of this area in monkeys (and also the corresponding region in dogs, cats, and rabbits) resulted in a peculiar torsion of the lip and nostril on the same side. This reaction was the same as that induced by direct application of strong or disagreeable odours to the nostril and was regarded as " evidently the outward or associated expression of excited olfactory sensation." In lower mammals it has been found by several investigators (for example, by Fox and others, 1944) that stimulation of the olfactory bulb is followed by conspicuous potential changes in the anterior piriform cortex. Some human cases provide further evidence that the primary olfactory cortex lies in the anterior hippocampal gyrus. Thus, Uyematsu (1921) has described two brains with unilateral destruction of the olfactory peduncle in which the resulting cellular atrophy was confined to the piriform area, "lateral to the gyrus circumambiens and anterior to the uncus proper," and this area appears to correspond to the temporal prepiriform cortex as delimited by us in the monkey brain.

\section{Summary}

1. The central connexions of the olfactory tracts have been determined experimentally in three macaque monkeys and two Guinea baboons by following with a silver method the degeneration of fibres and terminals which result from unilateral transection of the olfactory peduncle.

2. It was found that the lateral olfactory tract terminates mainly on the dorsal surface of the temporal lobe, in the superficial layer of the prepiriform cortex which forms the anterior part of the hippocampal gyrus. Some fibres of the tract terminate in the prepiriform cortex lying on the orbital surface of the frontal lobe (which is very restricted in primates), in the olfactory tubercle, and in the peri-amygdaloid area. The primary olfactory areas have been mapped out on the surface of the macaque brain (Fig. 17).

3. Only the cortico-medial group of nuclei of the amygdaloid complex receives fibres from the olfactory tracts, not the baso-lateral group. Olfactory fibres are bilaterally distributed by way of the anterior limb of the anterior commissure to the nucleus of the stria terminalis (the main part of which lies in the preoptic region), and to the central amygdaloid nucleus.

4. No olfactory fibres could be traced to the septum, the entorhinal area, or any part of the hippocampal formation in the lateral ventricle. A few fibres from the tract reach the superficial layer of the anterior hippocampal nucleus.

5. It is evident that in primates the general pattern of distribution of olfactory fibres is similar to that in lower mammals, although the primary olfactory areas have been displaced and have become relatively much reduced in the course of evolution.

We wish to express our thanks to Professor W. E. Le Gros Clark for his constant advice and constructive criticism, and also for performing one of the experimental operations, to Drs. W. H. Feindel and R. Hinshaw for performing the other operations, and to Mrs. M. Purves and Messrs. L. Cooper and P. Selwood for their valuable assistance in the preparation of the drawings, histological material, and low-power photomicrographs respectively. The investigation was undertaken while one of us (M. Meyer) was receiving a grant from the Medical Research Council, the other a grant from the Nuffield Committee for the Advancement of Medicine.

\section{REFERENCES}

Beck, E. (1934). J. Psychol. Neurol., 46, 193.

Brodal, A. (1947). Schweiz. med. Wschr., 37, 971.

Brodmann, K. (1909). "Vergleichende Lokalisationslehre der Grosshirninde." J. A. Barth., Leipzig, pp. 324.

Cajal, Ramón y (1901). Trab. Lab. Invest. biol., Univ. Madr., 1, 1. 
Cajal, Ramón y (1911). "Histologie du Système nerveux de l'Homme et des Vertébrés." Maloine, Paris. vol. 2. Crosby, E. C., and Humphrey, T. (1941). J. comp. Neurol., 74, 309.

Elliot Smith, G. (1909). Anat. Anz., 34, 200.

Fox, C. A., Fisher, R. R., and Desalva, S. J. (1948). J. comp. Neurol., 89, 245.

Fox, C. A., McKinley, W. A., and Magoun, H. W. (1944). J. Neurophysiol., 7, 1.

Hess, W. R., and Müller, H. R. (1946). Helv. Physiol. Acta., 4, 339.

Johnston, J. B. (1923). J. comp. Neurol., 35, 337.
Lauer, E. W. (1945). Ibid., 82, 215.

Le Gros Clark, W. E., and Meyer, M. (1947). Brain, 70, 304. Papez, J. W., and Aronson, L. R. (1934). Arch. Neurol.
Psychiat., Chicago, 32, 1.

Rose, J. E., and Woolsey, C. N. (1943). Fed. Proc. exp. Biol., 2, 42.

Rose, M. (1927a). J. Psychol. Neurol., 34, 1. (1927b). Ibid., 34, 261.

-(1928). Ibid., 37, 467.

Uyematsu, S. (1921). Arch. Neurol. Psychiat., Chicago $6,146$. 